\title{
Studies on Swelling Behavior of HPMC based Matrix Tablets by the Application of Digital Imaging Technique
}

\author{
Irin Dewan ${ }^{1}$, Md. Elias-Al-Mamun ${ }^{2}$ and Reza-ul J alil' \\ ${ }^{1}$ Department of Pharmacy, University of Asia Pacific, Dhanmondi, Dhaka-1209, Bangladesh \\ ${ }^{2}$ Department of Pharmaceutical Technology, Faculty of Pharmacy, University of Dhaka, \\ Dhaka-1000, Bangladesh
}

\begin{abstract}
The widespread application of hydrogels in a number of applications like control of microfluidic flow, development of muscle-like actuators, filtration/separation and drug delivery makes it important to properly understand these materials. Understanding hydrogel properties is also important from the standpoint of their similarity to many biological tissues. The degree of swelling as well as the rate of swelling of the hydrogel was studied through experiments using digital photography. First of all the matrix was prepared by blending properly weighed HPMC, Theophylline and excipients in a laboratory mixture for 10 minutes followed by compression with 5 ton force for 30 seconds. The study was designed to evaluate the effect of hydrophobic materials on HPMC matrix tablets in terms of in vitro swelling study. Different viscosity grades of HPMC i.e. 6- cps, 15-cps and 50-cps were used to prepare tablets along with various hydrophobic materials. Among the hydrophobic materials stearic acid (SA), cetyl alcohol (CA), bees wax (BW), glyceryl monostearate (GMS), cetostearyl alcohol (CSA) and stearyl alcohol (STA) were used. It was found that presence of hydrophobic ingredients in the HPMC hydrogel matrix substantially reduces swelling of the matrix. In this work, the extent of swelling of HPMC matrix was evaluated by using digital photography and counting the pixels with Photoshop software. Finally, it was shown from the experiment that the rate and extent of drug release is mainly governed by swelling rate of different HPMC viscosity grade used and the nature of hydrophobic materials included into the matrix system.
\end{abstract}

Key words: Swelling, Matrix, HPMC, Hydrogel, Photography.

\section{INTRODUCTION}

To ensure correct tablet disintegration and to overcome the harmful effect of some of the foregoing parameters, disintegrants are added during formulation. These are hydrophilic materials, which may be hydro-soluble and often tend to swell in contact with water.

Correspondence to: Dr. Reza-ul Jalil

Tel: +880-2-9120325; Fax: +880-2-8615583

E-mail: raju1559@yahoo.com

Dhaka Univ. J. Pharm. Sci. 7(2): 169-175, 2008 (December)
Different theories concerning disintegrants action mechanisms have led to a large number of investigations in recent years. Apart from disintegration caused by the mechanical action of a gas, which is liberated by effervescent, the theories advanced draw on adsorptivity, porosity, swelling, deformation and absorption mechanisms. Tablet disintegration, which is an absolute prerequisite for their speed of action, is a complex mechanism. Due to the complexity of their formula and their manufacturing method, tablets containing the same active ingredient may display widely varying 
disintegration time and speed of action. The action mechanism of disintegrations is not perfectly clear, although a very large number of hypotheses have been substantiated. ${ }^{1-5}$

New products are proposed today, designed to cause flash disintegrations, without slowing down dissolution or reducing the compressibility properties of the powders. The problem of tablet disintegration is hence a problem in permanent evaluation, and it is difficult to make any final declaration on the subject. Moreover, not only should disintegration be considered, but also the effect of disintegrants on the other characteristics of the tablets: compatibility, flow, compressibility, hardness, friability, colour, taste, and effect on storage. This means the subject has no bounds. ${ }^{6-8}$

Hence it is necessary to obtain a continuous disintegrate network as pointed out already by Patel and Hopponen (1966). It appears that the speed of disintegration is not only related to the water absorption capacity of a disintegrant, but also of its solubility. In fact, the combination of an insoluble diluents and a soluble disintegrant delays the diffusion of water in the capillaries.

For those tablets that are swallowed and are to break apart in the gastrointestinal tract, substances must be added to the formulation to oppose the binder and the compression forces needed to form the tablet. Several agents that act by different mechanisms may be used. ${ }^{9-12}$ The present study was designed to evaluate the degree swelling vs hydrophilic/hydrophobic matrix interaction.

\section{MATERIALS AND METHODS}

Chemicals. Theophylline (BDH), HPMC 6 cps, 15 cps \& 50 cps. (Samsung Fine Chemicals Co. Ltd. Korea), Steric acid (BDH), Cetyl alcohol (BDH), Bees wax (BDH), Glyceryl monostearate (BDH), Cetostearyl alcohol (BDH), Stearyl alcohol (BDH), Talc (BDH), Aerosil (FMC-USA), Mg-stearate (Malincot-USA).

Digital Photographic Equipments \& Software Used. Panasonic Digital Video Camera-3CCD,
Japan, V-Geer Digital Capture Card, Paint Shop Pro-7 for handing digital picture, Adobe Photoshop-7 for pixel count, Fine Pix for transferring digital picture from camera to computer.

Preparation of Matrix Tablets. Properly weighed HPMC, Theophylline and excipients were blended in a laboratory mixture for 10 minutes. Particular attention has been given to ensure thorough mixing and phase homogenization. The appropriate amount of the mixture was then compressed using a Perkin-Elmer laboratory hydraulic press equipped with a $13 \mathrm{~mm}$ flat punch and die set. The compression force and compression time were 5 ton and 30 seconds respectively. Before compression, the surfaces of the die and punch were lubricated with magnesium stearate. Finally matrix tablets were stored in airtight containers at room temperature for further study. The formulation compositions of different tablets are shown in the Table 1.

Swelling Study By Digital Imaging. Swelling study was conducted from the different tablets for 8 hours in petridishes. The study was conducted in distilled water at $37^{\circ} \mathrm{C}$. At every 1-hour interval digital snap of the different tablets were taken by a Panasonic Digital video camera (1.7 mega pixel). Finally the digital snaps of swelling tablets were transferred from camera to computer using Fine pix software. The camera setup and the sample petridish are shown in the Figure 1.

\section{RESULTS AND DISCUSSION}

Effect of Different Excipients on Swelling Properties of HPMC Tablets. The study was designed to evaluate the effect of hydrophobic materials on HPMC matrix tablets in terms of in vitro swelling study. Different viscosity grades of HPMC i.e. 6-cps, 15-cps and 50-cps were used to prepare tablets along with the hydrophobic materials. Among the hydrophobic materials stearic acid (SA), cetyl alcohol (CA), bees wax (BW), glyceryl monostearate (GMS), cetostearyl alcohol (CSA) and stearyl alcohol (STA) were used. Panasonic Digital video camera (1.7 mega pixel) was used for imaging. 
Table 1. Batch formulation of HPMC tablet with different hydrophobic matrices

\begin{tabular}{|c|c|c|c|c|c|c|c|}
\hline \multirow{3}{*}{ INGREDIENTS } & \multicolumn{7}{|c|}{ Tablets Prepared From HPMC 6 cps Viscosity Grade } \\
\hline & H06B & H06SA & H06CA & H06BW & H06GMS & H06CETA & H06STA \\
\hline & \multicolumn{7}{|c|}{ (g) } \\
\hline Theophylline & 0.2 & 0.2 & 0.2 & 0.2 & 0.2 & 0.2 & 0.2 \\
\hline HPMC-6cps & 2.2 & 2.0 & 2.0 & 2.0 & 2.0 & 2.0 & 2.0 \\
\hline Steric Acid & & 0.2 & & & & & \\
\hline Cetyl Alcohol & & & 0.2 & & & & \\
\hline Bees Wax & & & & 0.2 & & & \\
\hline Glyceryl monostearate & & & & & 0.2 & & \\
\hline Cetostearyl Alcohol & & & & & & 0.2 & \\
\hline Stearyl Alcohol & & & & & & & 0.2 \\
\hline Talc & 0.035 & 0.035 & 0.035 & 0.035 & 0.035 & 0.035 & 0.035 \\
\hline Mg- stearate & 0.035 & 0.035 & 0.035 & 0.035 & 0.035 & 0.035 & 0.035 \\
\hline Aerosil & 0.030 & 0.030 & 0.030 & 0.030 & 0.030 & 0.030 & 0.030 \\
\hline \multirow[t]{2}{*}{ Total } & 2.5 & 2.5 & 2.5 & 2.5 & 2.5 & 2.5 & 2.5 \\
\hline & \multicolumn{7}{|c|}{ Tablets Prepared From HPMC 15 cps Viscosity Grade } \\
\hline INGREDIENTS & H15B & H15SA & H15CA & H15BW & H15GMS & H15CETA & H15STA \\
\hline Theophylline & 0.2 & 0.2 & 0.2 & 0.2 & 0.2 & 0.2 & 0.2 \\
\hline HPMC-15cps & 2.2 & 2.0 & 2.0 & 2.0 & 2.0 & 2.0 & 2.0 \\
\hline Steric Acid & & 0.2 & & & & & \\
\hline Cetyl Alcohol & & & 0.2 & & & & \\
\hline Bees Wax & & & & 0.2 & & & \\
\hline Glyceryl monostearate & & & & & 0.2 & & \\
\hline Cetostearyl Alcohol & & & & & & 0.2 & \\
\hline Stearyl Alcohol & & & & & & & 0.2 \\
\hline Talc & 0.035 & 0.035 & 0.035 & 0.035 & 0.035 & 0.035 & 0.035 \\
\hline Mg-stearate & 0.035 & 0.035 & 0.035 & 0.035 & 0.035 & 0.035 & 0.035 \\
\hline Aerosil & 0.030 & 0.030 & 0.030 & 0.030 & 0.030 & 0.030 & 0.030 \\
\hline \multirow[t]{2}{*}{ Total } & 2.5 & 2.5 & 2.5 & 2.5 & 2.5 & 2.5 & 2.5 \\
\hline & \multicolumn{7}{|c|}{ Tablets Prepared From HPMC 50 cps Viscosity Grade } \\
\hline INGREDIENTS & H50B & H50SA & H50CA & H50BW & H50GMS & H50CETA & H50STA \\
\hline Theophylline & 0.2 & 0.2 & 0.2 & 0.2 & 0.2 & 0.2 & 0.2 \\
\hline HPMC-50cps & 2.2 & 2.0 & 2.0 & 2.0 & 2.0 & 2.0 & 2.0 \\
\hline Steric Acid & & 0.2 & & & & & \\
\hline Cetyl Alcohol & & & 0.2 & & & & \\
\hline Bees Wax & & & & 0.2 & & & \\
\hline Glyceryl monostearate & & & & & 0.2 & & \\
\hline Cetostearyl Alcohol & & & & & & 0.2 & \\
\hline Stearyl Alcohol & & & & & & & 0.2 \\
\hline Talc & 0.035 & 0.035 & 0.035 & 0.035 & 0.035 & 0.035 & 0.035 \\
\hline Mg-stearate & 0.035 & 0.035 & 0.035 & 0.035 & 0.035 & 0.035 & 0.035 \\
\hline Aerosil & 0.030 & 0.030 & 0.030 & 0.030 & 0.030 & 0.030 & 0.030 \\
\hline Total & 2.5 & 2.5 & 2.5 & 2.5 & 2.5 & 2.5 & 2.5 \\
\hline
\end{tabular}

A pixel is a unit-building component of a digital picture. Number of pixel determines the size of a picture. Hence, the number of pixels in a particular area is equivalent to the size of the area. Therefore, it is very quick and very accurate method of determining a particular area of a digital picture. ${ }^{13-14}$ The magic tool of the Adobe photoshop software only select the similar pixels connected to each other. When the white core of the tablet is selected by the magic tool, it selects only the un-swelled area, 
therefore, the amount of unswelled part with time is obtained by calculating the number of pixels in the area. In other words the amount of swelling is obtained by subtracting it from the original size. ${ }^{15-16}$
An example of the pixel area is shown in the Figure 2. In this picture the number of pixel at zero hour was found 5690, at three hour time 4850 and at six hour 2201, indicating the reduction in size with time.

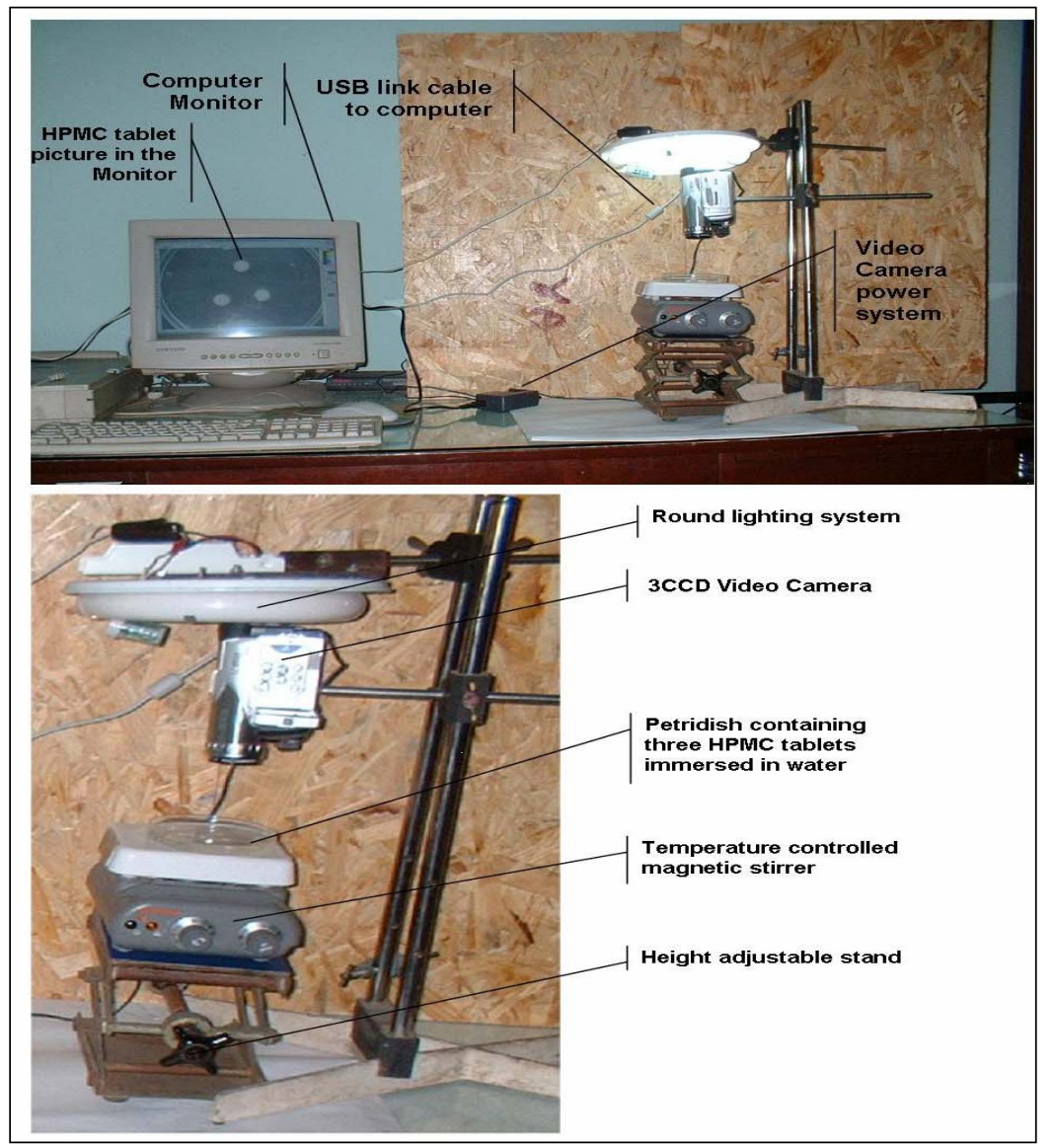

Figure 1. The video camera and computer setup along with the sampling petridish for snap shots of swelling of HPMC tablets.

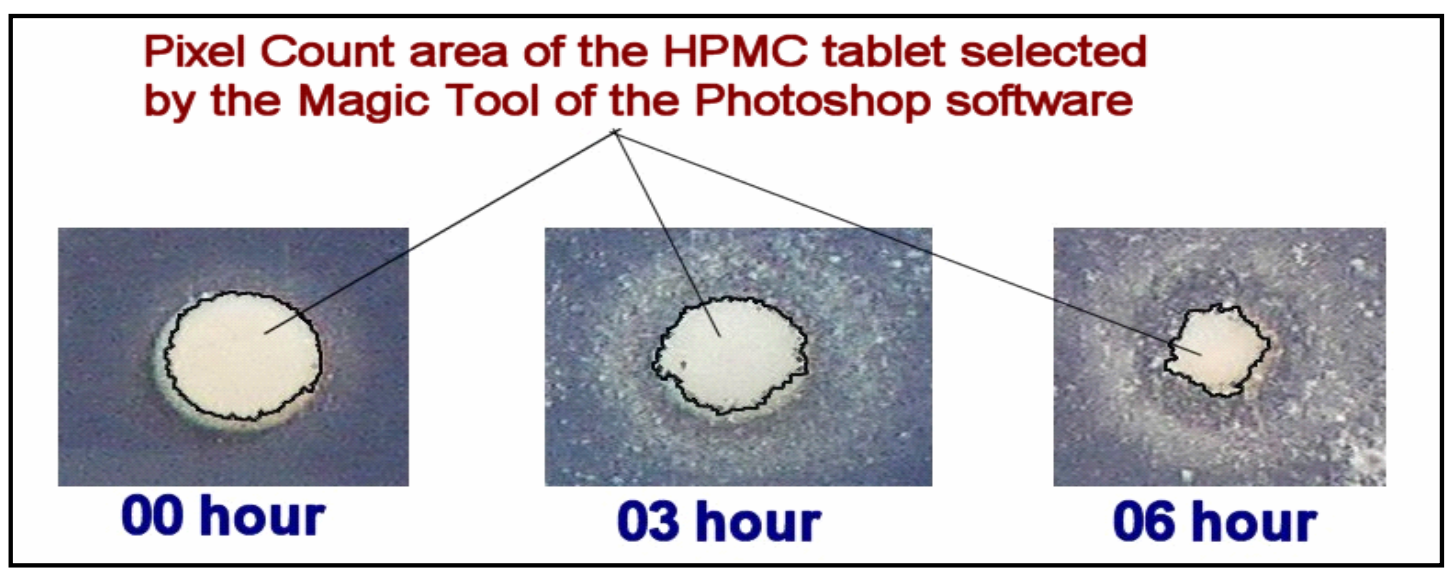

Figure 2. The picture showing the reduction of the white central core size of the HPMC tablet with time. The area within black boundary is selected by the magic tool of the Photoshop software, which also gives the number of pixels in the area. 


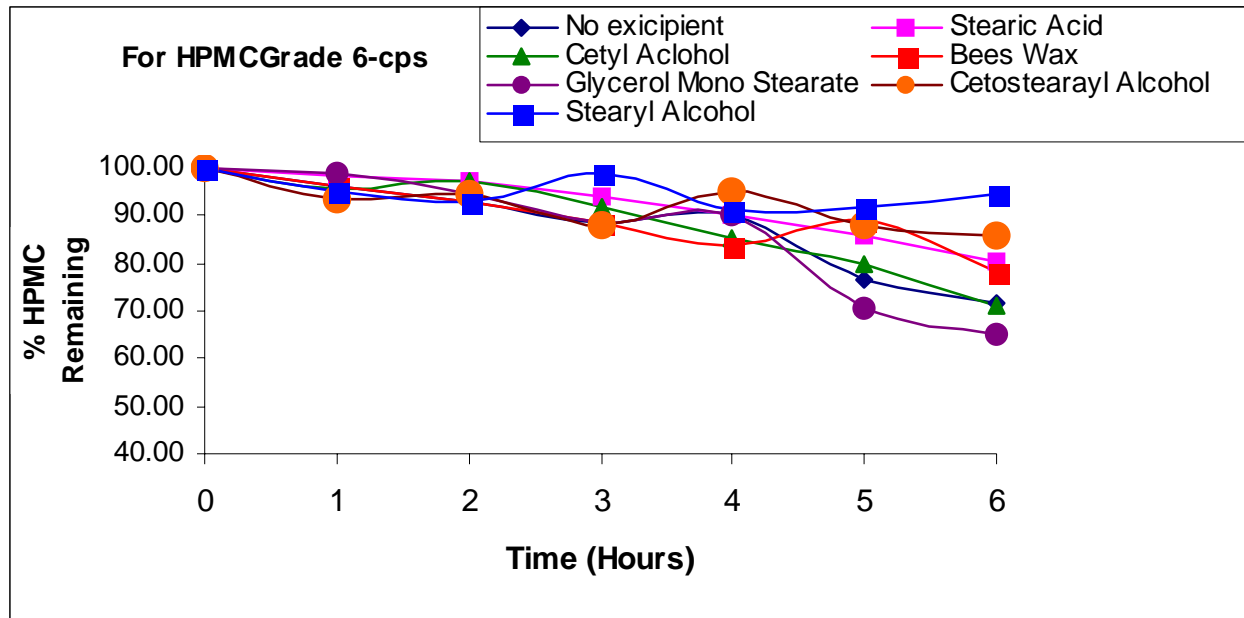

Figure 3. Percentage of HPMC (6cps) remaining from matrix tablet containing different excipients

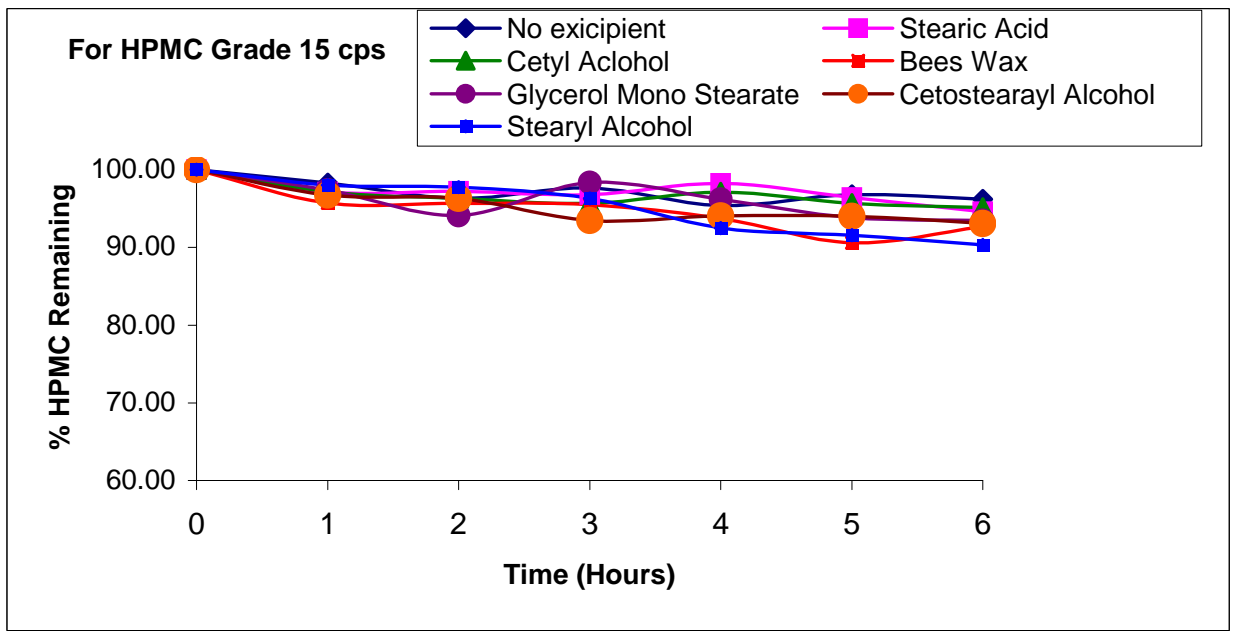

Figure 4. Percentage of HPMC (15cps) remaining from matrix tablet containing different excipients

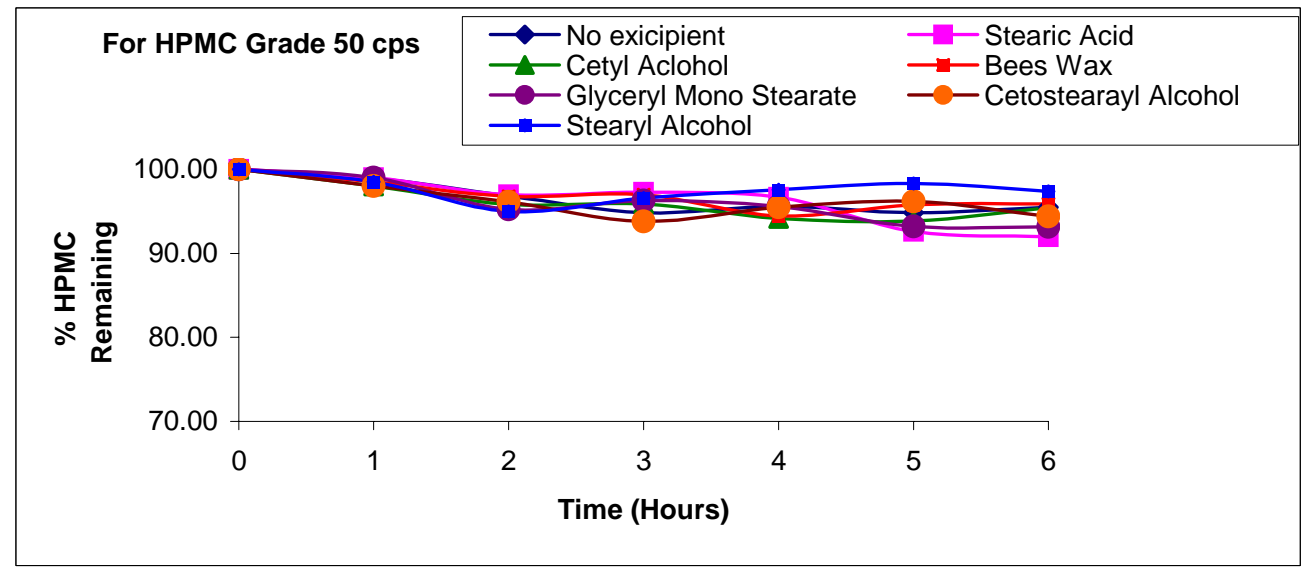

Figure 5. Percentage of HPMC (50cps) remaining from matrix tablet containing different excipients 


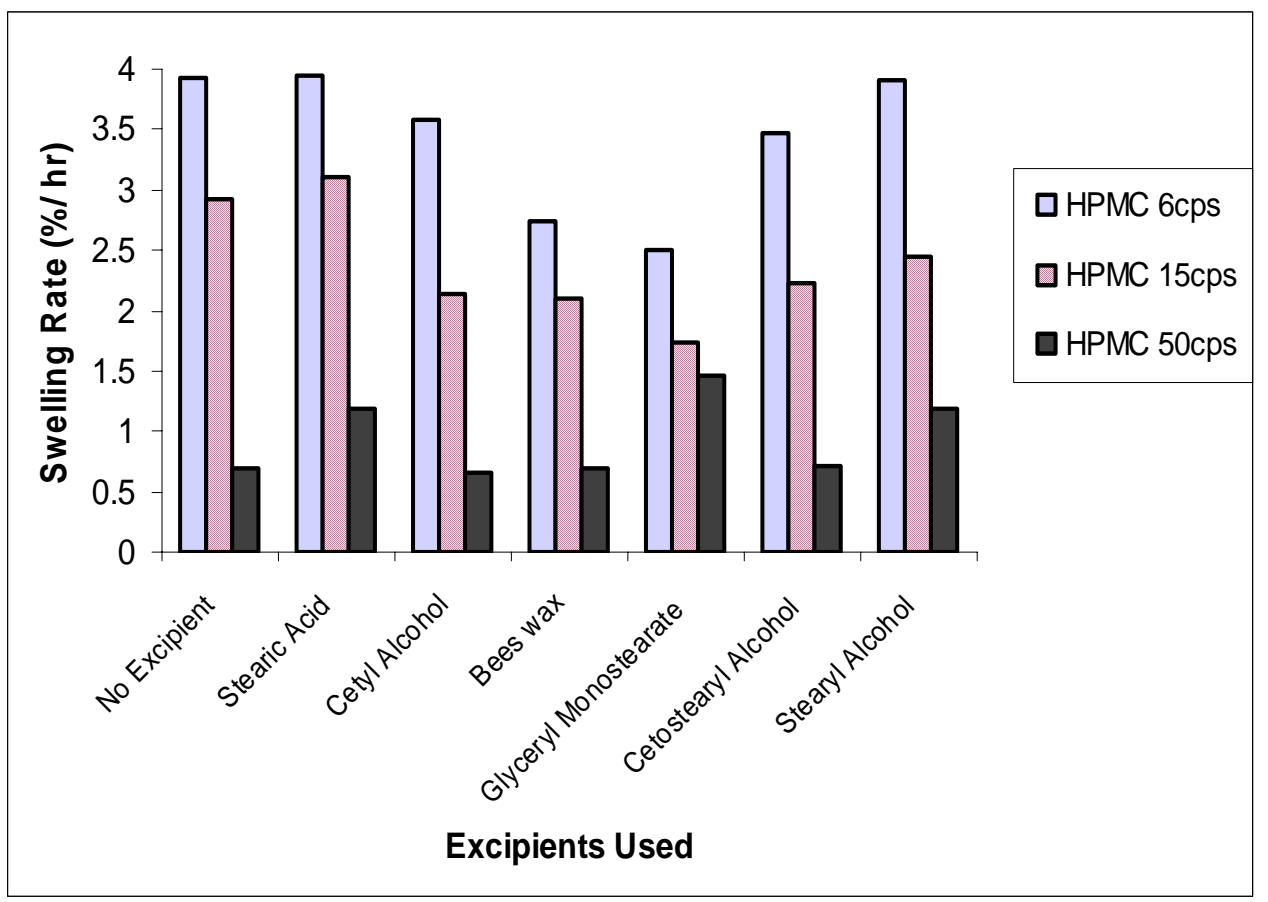

Figure 6. Effect on swelling rates of different excipients with different grades of HPMC.

The pixel count data, ${ }^{15-16}$ i.e. the \% HPMC remaining were then plotted against time. The pixel count at zero hour was taken as $100 \%$ and the swelling was increasing after hours with decreasing the pixel count to reduced percentage. The graphs are shown in Figure 3 to Figure 5. The rate of swelling was calculated from the trend line of the straight-line portion of the curve. The effects on swelling rates of the different tablets with different hydrophobic excipients in response to different grade of HPMC are presented in Figure 6.

Significant differences were found when different hydrophobic materials were incorporated into the HPMC matrix system. When stearic acid and stearyl alcohol were incorporated into HPMC 6-cps matrix, higher swelling rate was found. Because the carboxylic acid group (-COOH) of stearic acid and the hydroxyl group $(-\mathrm{OH})$ are hydrophilic in nature which facilitates the faster swelling of drug. All the other hydrophobic materials reduced the swelling rate after 8 hours than that from HPMC 6-cps matrix alone.
Swelling rate of HPMC 6-cps matrix tablets containing glyceryl monostearate (GMS) was reduced because the alcoholic group of GMS has been blocked by Stearate moiety. As a result the hydrophilicity of GMS has been reduced and hydrophobicity has been increased. The elevated hydrophobicity may be held responsible for reduced swelling rate. The order of swelling rate from HPMC 6-cps matrix tablets containing different hydrophobic polymers after 8 hours can be classed in the following way:

Stearic Acid $>$ Stearyl Alcohol $>$ Cetyl alcohol $>$ Cetostearyl Alcohol $>$ Bees Wax $>$ GMS .

When stearic acid, stearyl alcohol, cetostearyl alcohol were incorporated into HPMC-15 cps matrix tablets, the elevated swelling rate were observed. On the other hand swelling rate of bees wax and GMS were reduced when those were incorporated into the matrix, in this case, the order of swelling rate is as follows:

Stearic Acid > Stearyl Alcohol > Cetostearyl Alcohol $>$ Cetyl alcohol $>$ Bees Wax $>$ GMS. 
Here, again the hydrophobicity of different fatty materials governed the rate of release.

For HPMC 50-cps matrix tablets, highest swelling rate was found with GMS whereas lowest rate was found with bees wax. Inclusion of stearic acid increased the rate profile whereas inclusion of cetyl alcohol reduced the rate of release. The rate order is-

GMS > Stearic acid > Stearyl alcohol > Cetostearyl alcohol $>$ Bees wax $>$ Cetyl alcohol.

All this data showed that rate and extent of drug release is mainly governed by HPMC viscosity grade used and the nature of hydrophobic materials included into the matrix system.

\section{CONCLUSION}

The present study revealed that the swelling rate of HPMC matrix tablets prepared by direct compression method was mainly altered by viscosity grade and also incorporation of different hydrophobic polymers. The study also indicates that it is possible to modulate the rate and extent of drug release by judicious combination of hydrophobic material with the matrix-forming agent. The hydrophobic agents used in this formulation, being economic and available, will enable the formulator with higher degree of flexibility for optimizing the formulation and designing a desirable release characteristic of the active ingredient from the dosage form. At the same time it is concluded that the digital video imaging technique is a very fast and reliable tools in studying such swelling methods. Finally it is concluded that the availability of digital imaging technique could be a very reliable and useful tools in the process of product developments.

\section{REFERENCES}

1. A Medical Rationale, in Topical Drug Bioavailability, Bioequivalence, and Penetration, Shah VP, and Maibach HI (eds), New York, Plenum, pp. 17-68, 1993

2. Abstracts 218-224. 1992. The 19th International Meeting of The Controlled Release Society, July 26-31, Washington, DC.

3. Akiyama. Y, Yoshika. M, Horibe. H, Hirai. S, Kitamori. N and Tiguchi. H. 1993. Novel oral controlled-release microspheres using polyglycerol esters of fatty acids. J. Controlled Release 26, 1-10.

4. Alderman, D.A. 1984. A review of cellulose ethers in hydrophilic matrices for oral controlled release dosage forms. Int. J. Pharm. Technol. Prod. Manuf. 5, l 1-19.

5. Al-Shora, H., said, S. and Hammad, A.L. 1980. Sustained release from inert matrix II. Effect of Polyethylene glycols on theophylline release. Int. J. Pharmaceutics 7, 77-82.

6. Bergman, L.A. and Bendelin, F.J. 1965. Effects of concentration, aging and temperature on tablet disintegrants in a soluble direct-compression system, J. Pharm. Sci. 54, 445-447.

7. Berry, F.R. 1950. The preparation of Compresed Tablets I. The Measurement of the Rate of Disintegration of Compressed Tablets, Quart. J. Pharm. Pharmacol 12, 501510.

8. Buri, P. and Doelker, E. 1980. Formulation of extended released tablets II: hydrophilic matrics. Pharm. Acta Helv., 55, 189-197.

9. C. Wang. Y. Li, and Z. Hu. 1997. Swelling kinetics of polymer gels. Macromolecules 30, 727-4732.

10. Beebe. D.J., Moore, J. and Bauar, J.M. 2000. Functional hydrogel structures for autonomous flow control inside micro-fluidic channels. Nuture 404, 588-590.

11. Eichenbaum, G.M., Kiser, P.M., Simon, S.A. and Needham. D. 1998. $\mathrm{pH}$ and Ion triggered volume response of anionic hydrogel microspheres. Macromolecules 31, 5084-5093.

12. Chu, Y., Varanasi, P.P., Mcglade, M.J. and Varanasi. 1995. $\mathrm{pH}$-induced swelling kinetics of polyelectrolyte hydrogels. $J$. Appl. Polymer Sci. 8, 2161-2176.

13. http://en.wikipedia.org/wiki/Pixel.

14. http://mvh.sr.unh.edu/mvhinvestigations/pixel_count.htm

15. US Patent 7257264 - Image processing apparatus and method for compression-encoding image area information.

16. United States Patent 5805727-Image recognition method and apparatus. 